\title{
HPLC Method for the Determination Content of 5-HMF in Zishen Yutai Pill
}

\author{
Lin Shaozhu, Huang Minna, Liu Hui, Huang Jiawen, Jin Weijun, Wu Xiaosong* \\ Department of Pharmacy, The First Affiliated Hospital of Jinan University, Guangzhou, China \\ Email address: \\ 13556021313@163.com (Lin Shaozhu),418553103@qq.com (Huang Minna), liu_ui@hotmail.com (Liu Hui), \\ jinwj66@sina.com (Jin Weijun), eudaemoniay@139.com (Huang Jiawen), twxs2010@126.com (Wu Xiaosong) \\ ${ }^{*}$ Corresponding author
}

\section{To cite this article:}

Lin Shaozhu, Huang Minna, Liu Hui, Huang Jiawen, Jin Weijun, Wu Xiaosong. HPLC Method for the Determination Content of 5-HMF in Zishen Yutai Pill. International Journal of Chinese Medicine. Vol. 5, No. 1, 2021, pp. 14-17. doi: 10.11648/j.ijcm.20210501.13

Received: February 26, 2021; Accepted: March 17, 2021; Published: March 26, 2021

\begin{abstract}
Objective: During the preparation of Zishen Yutai Pills, 5-Hydroxymethylfurfural may be used. To establish a HPLC method for determination of 5-HMF in Zishen Yutai pill and to provide the potential quality control standard of it. Methods: RP-HPLC method was applied with the chromatographic condition as follows: the chromatographic column was Phenomenex Gemini C18 column $(250 \times 4.6 \mathrm{~mm}, 5 \mu \mathrm{m})$, methanol-water $(10: 90)$ as the mobile phrase, the flow rate was $0.8 \mathrm{~mL} / \mathrm{min}$, the detection wavelength was $284 \mathrm{~nm}$, the column temperature was $30^{\circ} \mathrm{C}$, and the injection volume was $10 \mu \mathrm{L}$. Results: The calibration curve was linear in the range of $2.16 \sim 43.27 \mu \mathrm{g} . \mathrm{mL}^{-1}$ for $5-\mathrm{HMF}\left(\mathrm{r}^{2}=0.9993\right)$. The RSD of the method repeatability was $0.97 \%(\mathrm{n}=6)$ and the average recovery was $99.4 \%(\mathrm{RSD}=0.58 \%)$. What's more, the specificity, precision and stability results were also good. Conclusion: The method for HPLC is not only sensitive and accurate. It is also easy to operate which can be used as the determination method for 5-HMF and has a guiding role for the quality control of the Zishen Yutai pill.
\end{abstract}

Keywords: HPLC, Zishen Yutai Pill, 5-HMF, Determination, Methodology

\section{Introduction}

Zishen Yutai pill (ZYP) is a traditional Chinese medicine compound preparation containing 15 Chinese herbal medicines. ZYP has rich clinical experience in treating various diseases such as infertility and miscarriage [1]. Recently, it has been reported that it can enhance the receptivity of the endometrium, prevent re-abortion, and enhance the spleen and kidney function. Similarly, it is an effective medicine for the treatment of menstrual disorders with luteal phase deficiency, asthenospermia and spleen and kidney deficiency type infertility $[2,3]$.

The main ingredients of ZYP are Codonopsis, Polygonum multiflorum, Eucommia, Cuscuta, Rehmannia glutinosa, etc. According to the literature, the 5-HMF content of Rehmannia glutinosa after processing will increase about 20 times. Honey, as a binder for ZYP will cause monosaccharides such as fructose to be heated to remove tri-molecular water to form 5-HMF, which increases the content of monosaccharides such as fructose. There are related literature reports that the daily allowable intake of 5-hydroxymethyl furfural is $30-60 \mathrm{mg}$, and foreign literature reports that the intake of 5-HMF is $1.6-150 \mathrm{mg} / \mathrm{person} /$ day. At present, there is a lot of controversy about 5-HMF discussion. According to reports in the literature, larger doses can damage human striated muscle and internal organs [4]. It is neurotoxic and can combine with human proteins to produce accumulated poisoning [5] and abnormal growth of colonic sac [6], which seriously affects the health of patients taking the drug. The "Chinese Pharmacopoeia" (2010 edition) [7] has clearly listed 5-HMF as an impurity item in the quality standards for glucose-containing injections for monitoring. The existing literature research focuses on the determination of 5-hydroxymethylfurfural content, and seldom involves the determination of 5-hydroxymethylfurfural in ZYP [8].

This article will focus on this issue. The establishment of a high performance liquid chromatography determination method aims to provide a reference for the quality control of the drug. 


\section{Methods}

\subsection{Instruments}

Agilent 1260 high-performance liquid chromatograph, Agilent 1260 G4212B PDA detector, American Agilent company; Sartorius BT125D electronic balance, 1/100,000, Sartorius, Germany; Sartorius BSA124S electronic balance, 1/10,000, Sartorius, Germany Si company; H/T16MM desktop high-speed centrifuge, Hunan Hexi Instrument Equipment Co., Ltd.; KQ-300DE CNC ultrasonic cleaning instrument, Kunshan Ultrasonic Instrument Co., Ltd.; UPT-IV-20L ultrapure water system, Chengdu Yuyou Technology Co., Ltd.

\subsection{Main Reagents}

5-hydroxymethyl furfural reference substance was purchased from National institues for food and drug control (Lot Number: 111626-201509) and ZYP was from Guangzhou Zhongyi Pharmaceutical Company (Lot Number: S00033, T00058, T00061, Guangzhou, China).

\subsection{High-performance Liquid Chromatographic Analysis}

Chromatographic column: Phenomenex Gemini C18 column $(250 \mathrm{~mm} \times 4.6 \mathrm{~mm}, 5 \mu \mathrm{m})$; mobile phase is methanol-water (10:90); volume flow rate is $0.8 \mathrm{~mL} / \mathrm{min}$; detection wavelength is $284 \mathrm{~nm}$; column temperature is $30^{\circ} \mathrm{C}$; sample volume is $10 \mu \mathrm{L}$.

\subsection{Preparation of Standard Solutions}

A standard stock solution of 5-HMF was prepared by dissolving $11.06 \mathrm{mg}$ of 5-HMF reference substance, place it in a $10 \mathrm{~mL}$ brown measuring flask, add methanol to dissolve, dilute to the mark, and shake well. Precisely pipet $5 \mathrm{~mL}$, place it in a $25 \mathrm{~mL}$ brown volumetric flask, add methanol to dilute to the mark, shake well, and get a mass concentration of $0.2212 \mathrm{mg} \cdot \mathrm{ml}^{-1}$ reference substance stock solution.

\subsection{Preparation of Sample Solutions}

The powder of ZYP samples quantitatively $(0.2 \mathrm{~g})$ transferred into round-bottomed flask and extracted with $25 \mathrm{ml}$ of mobile phase and weighed after well mixed, and then placed $12 \mathrm{~h}$, Ultrasounded for $30 \mathrm{~min}(300 \mathrm{~W}, 40 \mathrm{kHz})$, and reweighed with deionized water added to make up the weight before centrifuged at $10000 \mathrm{r} \cdot \mathrm{min}$ for $30 \mathrm{~min}$, then taken the supernatant as the test solution.

\subsection{Preparation of Negative Blank Solution}

The Chinese medicinal materials in relevant prescriptions were taken and the negative samples lacking 5-HMF were prepared according to the preparation process of ZYP sample, and the negative blank solution was prepared according to the item "2.5".

\section{Results}

\subsection{Specificity Test}

Under the chromatographic condition of "2.3", the reference solution, test solution and negative blank solution were sampled with $10 \mu \mathrm{l}$ each, and the chromatogram was recorded to observe whether other components interfered with the sample peak.

The results showed that at the corresponding position of 5-HMF chromatographic peak, the test product had the same retention peak with the retention time of about $10.96 \mathrm{~min}$, while the negative sample solution had no absorption and no interference at this peak position.

\subsection{Linear Relation Investigation}

The reserve solution of reference was accurately removed into $10 \mathrm{~mL}$ volumetric flask $0.1,0.3,0.5,0.8,1.0,1.5$ and 2.0 $\mathrm{mL}$, diluted with methanol to scale, shaken well, and injected $10 \mu \mathrm{L}$. Linear regression was performed with the peak area $(\mathrm{Y})$ as the ordinate and concentration $(\mathrm{X})$ as the abscess coordinate The regression equation, correlation coefficient $(\mathrm{R})$ and linear range were $\mathrm{Y}=1.159 \times 107 \mathrm{X}-1.665 \times 106(\mathrm{R} 2=0.9993)$, and the linear range was $2.16 \sim 43.26 \mu \mathrm{g} \cdot \mathrm{ml}^{-1}$.

\subsection{Precision Test}

$10 \mu \mathrm{L}$ of reference solution was absorbed accurately, and the sample was repeated for 6 times according to the chromatographic conditions under "2.3". The peak area was recorded and the RSD was calculated. The results showed an RSD of $0.67 \%(n=6)$. The precision of the instrument is good.

\subsection{Repeatability Test}

Samples of ZYP (Lot Number. S00033) were taken and ground into fine powder. Six samples of the test solution were prepared in parallel according to item " 2.5 ", and samples were injected under item "2.3" for detection. The results showed that the average content of 5-HFM was $0.21 \%$ and the RSD was $0.97 \%(n=6)$. The results showed that the method had good repeatability.

Table 1. Determination results of sample recovery.

\begin{tabular}{|c|c|c|c|c|c|c|c|}
\hline composition & Sample weight /g & Content in sample /mg & $\begin{array}{l}\text { Amount } \\
\text { added /mg }\end{array}$ & $\begin{array}{l}\text { Measured } \\
\text { quantity /mg }\end{array}$ & recovery $/ \%$ & $\begin{array}{l}\text { Average } \\
\text { recovery } / \%\end{array}$ & RSD $/ \%$ \\
\hline \multirow{6}{*}{ 5-HFM } & 0.1012 & 0.2156 & 0.2163 & 0.4314 & 99.9 & \multirow{6}{*}{99.4} & \multirow{6}{*}{0.58} \\
\hline & 0.0996 & 0.2122 & 0.2163 & 0.4251 & 99.2 & & \\
\hline & 0.0999 & 0.2127 & 0.2163 & 0.4234 & 98.7 & & \\
\hline & 0.1008 & 0.2146 & 0.2163 & 0.4318 & 100.2 & & \\
\hline & 0.1001 & 0.2133 & 0.2163 & 0.4249 & 98.9 & & \\
\hline & 0.0995 & 0.2120 & 0.2163 & 0.4262 & 99.5 & & \\
\hline
\end{tabular}




\subsection{Recovery Test}

As shown in Table 1, six copies of ZYP (Lot Number. S00033) with known content were accurately weighed, $0.1 \mathrm{~g}$ for each, and $1 \mathrm{~mL}$ of reserve solution of reference substance with concentration of $0.2212 \mathrm{mg} \cdot \mathrm{mL}^{-1}$ was added to each, and the test product solution was prepared according to the method of "2.5", and the chromatographic conditions under " 2.3 " were determined. The calculated recoveries were $98.7 \%-100.2 \%$ with $\mathrm{RSD}<2 \%$.

\subsection{Stability Test}

One portion of the test solution prepared according to " 2.5 " was injected for detection at $0 \mathrm{~h}, 1 \mathrm{~h}, 3 \mathrm{~h}, 7 \mathrm{~h}$ and $9 \mathrm{~h}$ respectively. The peak area was recorded, and the calculated RSD was $0.74 \%$. The results showed that the test solution was basically stable within $9 \mathrm{~h}$.

\subsection{Sample Determination}

The samples of ZYP with Lot Number S00033, T00058 and T00061 were prepared in parallel according to the method of "2.5" and the chromatographic conditions of "2.3" were determined. The content of 5-HMF in three batches of ZYP was calculated. The results are shown in Table 2.

Table 2. Determination of 5-HMF in 3 batches of samples.

\begin{tabular}{ll}
\hline Lot Number & Content in sample $/\left(\mathbf{m g} \cdot \mathbf{g}^{-1}\right)$ \\
\hline S00033 & 2.13 \\
T00058 & 3.07 \\
T00061 & 2.80 \\
\hline
\end{tabular}

\section{Discuss}

In recent years, the safety of drugs has attracted more and more attention. According to the statistics of the national adverse drug reaction monitoring network, the number of adverse drug reactions time showed an increasing trend from 1990 to 2020. Therefore, effective control of harmful components in Chinese patent medicine has become the focus and hot spot of current research. Studies have shown that 5-HMF has a variety of pharmacological activities, but its obvious toxicity at a certain dose cannot be ignored. Therefore, 5-HMF is defined as a substance with dual effects of pharmacological activity and toxicity.[9-10].

5 -HFM is mainly an aldehyde compound produced by the dehydration of monosaccharide compounds such as glucose under high temperature or weak acid conditions. Its chemical properties are relatively active, with high reactivity and polymerization ability [11]. It can form a variety of derivatives through oxidation, hydrogenation and condensation reactions. Therefore, it has low cytotoxicity and low mutagenicity to human cells [12].

As a sugar degradation product, 5-HFM has many factors affecting its content due to its poor stability. In recent years, there are many reports on the determination of 5-HMF in Chinese patent medicine preparations and Chinese herbal pieces. In this study, 5- HFM in ZYP was used as the research object, and a method for the determination of 5- HFM by HPLC was established. Through literature review and previous experiments, it was determined that the extraction efficiency of ZYP was the highest when $10 \%$ methanol was used for $30 \mathrm{~min}$. The absorption of 5-HMF was the highest at $284 \mathrm{~nm}[13,14]$. Therefore, the extraction method and detection wavelength were determined. From the results of methodological investigation, 5-HMF showed a good linear relationship with the peak area in the concentration range of $0.0200-0.4002 \mu \mathrm{g}$, the correlation coefficient $\mathrm{R} 2=0.9993$, and the results of specificity, precision, repeatability, average recovery, durability and stability test were qualified. HPLC method is not only sensitive and accurate, but also easy to operate. It can be used for the determination of 5-HMF, and has a certain guiding role for the quality control of ZYP [15]. The components of traditional Chinese medicine compound preparation are complex, which can not be evaluated and analyzed by one or several index components. It is not comprehensive, because it is often a comprehensive process, so it needs further study.

At present, most studies on the pharmacological activity of 5-HMF are limited to the cellular level. Whether it is ultimately harmful to the human body depends on different exposure routes, exposure doses and frequencies. It is necessary to conduct in-depth toxicity and limit studies on 5-HMF and its related products, which has practical and guiding significance for the quality control and safety application of foods and drugs containing 5-HMF.

\section{Conclusion}

The components of traditional Chinese medicine compound preparation are complex, which can not be evaluated and analyzed by only one or several index components. It is not comprehensive, because it is often a comprehensive process. However, ZYP is suitable for special population and may have potential harm. The author thinks that it is of certain significance to establish a limit test for 5-HMF or as an index in the quality control of ZYP to ensure its quality and clinical medication safety.

\section{References}

[1] Ying S and Fengjuan H. (2020). Mechanisms of Zishen Yutai Pills Intervention with Kidney Yang as Mediator in the Treatment of Diminished Ovarian Reserve. Jilin Journal of Chinese Medicine 40: 1117-1120.

[2] Mei W, Yuexin W, Yingru W, Ning L, Min F, Wenzhi Z, Ran J, Xuliang $\mathrm{H}$ and Yan N. (2020). Research progress on content changes of 5-hydroxymethyl furfural during processing of Chinese medicine and it's pharmacological effects. Drug Evaluation Research 43: 2319-2327.

[3] Juncheng W. (2020). Determination of 5-hydroxymethyl Furfural in Breviscapine and Glucose Injection by HPLC and Its Limit Setting. Asia-Pacific Traditional Medicine 16: 47-50. 
[4] Ling L and Naijiang C. (2020). Determination of Genotoxic Impurity 5-Hydroxymethylfurfural in Calcium Gluconate Oral Solution by HPLC. China Pharmacist 23: 360-362.

[5] Fu-Hai S. (2020). Preparation of 5-hydroxymethylfurfural in honey. Journal of Food Safety \& Quality 11: 6450-6455.

[6] Kong F, Lee BH and Wei K. (2019). 5-Hydroxymethylfurfural Mitigates Lipopolysaccharide-Stimulated Inflammation via Suppression of MAPK, NF- $\mathrm{KB}$ and mTOR Activation in RAW 264.7 Cells. Molecules 24: 275.

[7] Jia L, Yichao L, Yang Y and Feng-juan H. (2019). Clinical observation of the effect of zishenyufeteng pill on endometrial volume of IVF-ET patients with repeated failure of kidney deficiency. Journal of Tianjin University of Traditional Chinese Medicine 38.

[8] Qi-guo HYYL. (2019). Study on the 5-hydroxymethylfurfural Constituents of Jiu Hua Rhizoma Polygnoati in"Nine-steam-nine-bask"Processing. Journal of Dezhou University: 29-32.

[9] Anburajan P, Pugazhendhi A, Park J, Sivagurunathan P, Kumar $G$ and Kim S. (2018). Effect of 5-hydroxymethylfurfural (5-HMF) on high-rate continuous biohydrogen production from galactose. Bioresour Technol: 1197-1200.

[10] Lin L, Ni L, Yahao L, Encan L, Xiaoyu F, Sheng L, Qingce Z,
Jiuming $\mathrm{H}$ and Hongtao J. (2018). Acute toxicity and cytotoxicity of 5-hydroxymethyl furfural.: 205-209.

[11] Zhuo Pan, He-Xin Shao, Liu T, Lu X and Fan X. (2017). Research progress of 5-hydroxymethylfurfural, a safety-related substance in traditional Chinese medicine injections. Zhongguo Zhong Yao Za Zhi 42: 1842-1846.

[12] Tingfeng W, Yuantang X and Danru Z. (2017). Efficacy and Safety Analysis of Zishen Yutai Pills Combined with Progesterone in the Treatment of Threatened Abortion. Journal of Hunan University of Chinese Medicine 39: 907-912.

[13] Zhou L, Huang Q, Wang R, Zhou J, Ma A, Chong L, Wu Y, Wang Y, Xu L, Chen Y, Jia Y, Gui B and Sun Z. (2016). Reproductive Toxicity of Zishen Yutai Pill in Rats: The Fertility and Early Embryonic Development Study (Segment I). Evid Based Complement Alternat Med 2016: 3175902.

[14] Ke-Han P and Shi-Yi O. (2016). Formation and mitigation of 5 -hydroxymethylfurfural in foods. Journal of Food Safety \& Quality 7: 251-256.

[15] Gao Q, Han L, Li X, Cai X and Li X. (2015). Traditional Chinese Medicine, the Zishen Yutai Pill, Ameliorates Precocious Endometrial Maturation Induced by Controlled Ovarian Hyperstimulation and Improves Uterine Receptivity via Upregulation of HOXA10. Evidence-based complementary and alternative medicine 2015: 317510-317586. 